\title{
sciendo
}

\section{PREVALENCE AND GENETIC DIVERSITY OF C. JEJUNI ISOLATED FROM BROILERS AND THEIR ENVIRONMENT USING flaA-RFLP TYPING AND MLST ANALYSIS}

\author{
Viktorija Lègaudaitė Lydekaitienè ${ }^{1 *}$, E. Kudirkienè² \\ ${ }^{1}$ Department of Food Safety and Quality, Lithuanian University of Health Sciences, \\ Veterinary Academy, Tilžès 18, 47181, Kaunas, Lithuania \\ ${ }^{2}$ Department of Veterinary Disease Biology, University of Copenhagen, Stigbøjlen 4 , \\ 1870 Frederiksberg C, Denmark \\ •Corresponding author: viktlega0908@kmu.lt
}

\begin{abstract}
Campylobacter is highly diverse genetically and also undergoes frequent intraspecific recombination. A major source of campylobacteriosis, which is transmitted to humans is found in poultry. The assessment of the genetic diversity among Campylobacter population is critical to our understanding of the epidemiology. The genetic diversity of Campylobacter jejuni isolates in broilers and their environment were investigated by flaA-restriction fragment length polymorphism (RFLP) and multilocus sequence typing (MLST). The study revealed that $92.3 \%$ of the examined broiler flocks were contaminated with Campylobacter spp. A total number of 35 different flaA types defined by $f l a \mathrm{~A}-\mathrm{RFLP}$ were found in $448 \mathrm{C}$. jejuni isolates originated from broilers, litter, puddles, zones, anteroom and wild birds. The most dominant flaA type was XXV. MLST defined 20 sequence types (STs) belonging to 10 clonal complexes (CCs). Among all the STs 9 isolates (15\%) were consigned to 2 different STs (ST-7413 and ST-4800), which could not be assigned. The most common CCs were ST-21 and ST-179. The ST-21 CC was common in broilers and environment (puddle water and concentric zones) and the ST-179 CC was specific to wild birds, but also was found in puddle water and concentric zones.
\end{abstract}

Key words: Campylobacter jejuni, poultry, MLST, RFLP, campylobacteriosis

Campylobacter jejuni continues to be the most common case of bacterial gastroenteritis in the developed world (EFSA, 2015). In the European Union the incidence of campylobacteriosis is 64.8 per 100,000 population (EFSA, 2015). In 2013 in Lithuania campylobacteriosis was the most prevalent food-borne zoonosis in humans with the incidence of 38.3 per 100,000 population (EFSA, 2015) and it was still growing till 2009. Compared with the Baltic States, in Lithuania campylobacteriosis incidence is higher than in Estonia $(28.9$ per 100,000$)$ or Latvia $(0.4 / 100,000)$ 
(EFSA, 2015). However, in neighboring Poland the incidence of this disease is only 1.4 per 100,000 (EFSA, 2015).

The risk factors of Campylobacter infections include the consumption of undercooked meat, contaminated food, water, raw milk and also a direct contact with pets, farm animals and children swimming in lakes and traveling abroad (Jakopanec et al., 2008; Richardson et al., 2007; Ellis-Iversen et al., 2012). But poultry is still a major source of this organism (EFSA, 2015). The investigations in Lithuania showed that broilers' caecal samples had a prevalence of $80.95 \%$ (Kudirkienè et al., 2010), broiler wings and drumsticks - 46.55\% (Bunevičienè et al., 2010) and cattle - 78.5\% (Ramonaite et al., 2013).

Molecular typing has advanced many epidemiological studies, including the identification of infections of food-borne outbreaks. The molecular epidemiological survey of $C$. jejuni is critical to understand the sources and routes of transmission (Levesque et al., 2008), as well as to develop digression strategies to reduce the incidence of campylobacteriosis (Moffatt et al., 2010). Thus, several methods including MLST (multilocus sequence typing) and flaA-RFLP (the restriction fragment length polymorphism analysis of the flagellin-A gene) have been used for epidemiological studies. The MLST has become the gold standard method for studying $C$. jejuni population (Korczak et al., 2009; Larsen et al., 2012). Some $C$. jejuni genotypes isolated from the surrounding environment of certain animals and chickens are found, these vehicles and reservoirs could be a source of contamination for broilers (Ridley et al., 2011).

Due to the increasing number of campylobacteriosis cases in Lithuania and in other countries, it is important to clarify $C$. jejuni population structure and phylogenetic relationships among the samples isolated from broilers and their environment. This study investigated $C$. jejuni population by MLST and flaA-RFLP among broilers and their environment over the period of two years. The aim of this study was to determine genetic diversity of $C$. jejuni and investigate the potential relationships between broilers and their environment.

\section{Material and methods}

The largest poultry factory in Lithuania has been chosen for the survey. The samples were taken from study house and additional houses during two years' period. The study house is the main poultry house, which was tested from empty house period till the end of broilers growing period. The additional houses were three poultry houses which were near or in front of the study house. In these three houses Campylobacter migration was observed.

\section{Flock sampling}

The samples from flock were taken once a week from litter (before a broiler placement day and during the raising period of the entire flock). The samples were collected from 13 broiler flocks. To sample a broiler house, one pair of disposa- 
ble fabric overshoes (boot socks) was worn over rubber boots as the collector and was used to walk a minimum of 100 meters inside the house. Before use, the boot socks were pre-moistened with sterile physiological saline maximum recovery broth (610077, Liofilchem, Italy) in order to allow the maximum uptake of Campylobacter cells from the litter. The boot socks were placed into sterile plastic bag and transported to the laboratory. In case a boot sock was considered positive by PCR, 10 fecal swabs from broiler cloaca were taken to confirm positivity once a week till the first depopulation event. The swabs were placed into tubes with $10 \mathrm{ml}$ sterile modified Exeter broth, which was prepared from Bolton broth (CM985, Oxoid, England) with Campylobacter growth (SV61, Mast Diagnostics, Merseyside U.K), Campylobacter enrichment (Exeter) (SV59 Mast Diagnostics, Merseyside UK) supplements, and $1 \%$ of the lacked horse blood as described previously (Williams et al., 2012). Before slaughter 10 fecal samples were taken for the detection of total Campylobacter spp. number and were placed into sterile plastic bags.

\section{Environmental sampling}

The samples from the environment were also taken weekly. We took the samples from the zones around the poultry farm, puddles, tap water, flies and feces from rats, wild animals and birds. The environment around the poultry farm was divided into 5 zones $(1 \mathrm{~m}, 40 \mathrm{~m}, 60 \mathrm{~m}, 80 \mathrm{~m}$ and $100 \mathrm{~m})$. The samples were taken from the boot socks as described above. The samples from the puddles were taken in sterile $100 \mathrm{ml}$ bottles all year round except winter. The tap water samples were taken from a study house and additional houses also in sterile $100 \mathrm{ml}$ bottles, when the broiler age was 20-25 days. Before taking the samples the tap was sterilized and the water was lowered from 3 to 5 minutes. The feces of wild animals, rats and birds were put into sterile bags. Flies were taken with a sterile pincer and put into sterile bags too.

\section{Isolation procedure and identification}

Two hundred $\mathrm{ml}$ of sterile saline maximum recovery broth (610077, Liofilchem, Italy) was added into the plastic bags with the boot socks taken from the litter and zones and was palpated by hand to release the fecal material. The procedure was used according to the technique of Merga et al. Thus, $10 \mu \mathrm{l}$ from the same saline with boot socks was streaked onto the Campylobacter blood free medium base (mCCDA) (610130, Liofilchem, Italy) with mCCDA Selective Supplement (81037, Liofilchem, Italy). The plates were incubated in a micro-aerophilic atmosphere $(5 \%$ oxygen, $10 \%$ carbon dioxide, $85 \%$ nitrogen) generated by Campygen (CN25; Oxoid, UK) at $37^{\circ} \mathrm{C}$ for $48 \mathrm{~h}$. After the incubation, the colonies suspected of Campylobacter were obtained from each plate, examined under a microscope and further purified on the blood agar plates (610188, Liofilchem, Italy) supplemented with 5\% lacked horse blood (HBL100, E\&O Laboratories, Scotland), and incubated at $37^{\circ} \mathrm{C}$ for $48 \mathrm{~h}$ in the microaerophilic atmosphere. The subcultured isolates were subsequently stored at $-80^{\circ} \mathrm{C}$ in Brain Heart Infusion Broth (BHI) (610008, Liofilchem, Italy) with $30 \%$ glycerol (REACHEM, Slovakia) until further use.

Broiler cloacal samples were collected using sterile cotton swabs and directly plated on mCCDA. Plates were incubated in a micro-aerophilic atmosphere at $37^{\circ} \mathrm{C}$ 
for $48 \mathrm{~h}$. The fecal samples from the broilers, which were used for the detection of total Campylobacter spp. and also the feces of wild animals, birds and rats were poured on with the modified Exeter broth with proportion 1:10 (1 g of fecal with $10 \mathrm{ml}$ of Exeter broth) and put into the BagMixer for 1 minute. For broiler fecal samples the decimal dilutions up to $10^{-10}$ were prepared. The Campylobacter counts $(\mathrm{cfu} / \mathrm{g})$ of the broiler fecal samples were calculated according to ISO 10272-2:2006. One hundred $\mu 1$ were taken from every dilution and put onto mCCDA agar. Ten $\mu 1$ from the saline with the feces of wild animals, birds and rats was also streaked onto mCCDA agar. All the plates were incubated in a micro-aerophilic atmosphere at $37^{\circ} \mathrm{C}$ for $48 \mathrm{~h}$. Each fly was macerated in a sterile mortar, suspended in $2 \mathrm{ml}$ of $0.9 \%$ saline. The mixture was centrifuged at $14,000 \mathrm{rpm}$ for $7 \mathrm{~min}$. The pellet was resuspended in $2 \mathrm{ml}$ of Exeter broth and vortexed before the incubation for the enrichment at $37^{\circ} \mathrm{C}$ for $24 \mathrm{~h}$. After the enrichment, the tube was again centrifuged and $10 \mu \mathrm{lwas}$ streaked onto mCCDA agar and incubated at $37^{\circ} \mathrm{C}$ for $48 \mathrm{~h}$ in microaerophilic atmosphere. The identification and purification of Campylobacter isolates were further performed as described above.

Additionally, a selective enrichment procedure was performed for each sample to detect the low numbers of Campylobacter spp. For this purpose, broiler samples swabs were placed into $10 \mathrm{ml}$ modified Exeter broth. Also $1 \mathrm{ml}$ of clear fluid from the saline with boot socks from litter and zones was transferred into $10 \mathrm{ml}$ of Exeter broth for the enrichment. This Exeter broth was prepared from Bolton broth (CM985, Oxoid, England) with Campylobacter growth (SV61, Mast Diagnostics, Merseyside UK), Campylobacter enrichment (Exeter) (SV59 Mast Diagnostics, Merseyside UK) supplements, and 1\% of the lacked horse blood as described previously (Williams et al., 2012). The enrichment tubes were incubated into micro-aerophilic atmosphere at $37^{\circ} \mathrm{C}$ for $48 \mathrm{~h}$. After the incubation $10 \mu \mathrm{l}$ of the broth from other samples (boot socks, feces, puddles, tap water), was streaked onto mCCDA plates. The identification and purification of Campylobacter isolates were further performed as described above.

\section{DNA extraction and multiplex PCR}

PureLink ${ }^{\circledR}$ Genomic DNA Kit (Life Technologies, Lithuania) was used for the DNA isolation direct from boot socks samples and the presence of Campylobacter spp. was confirmed by using PCR assay by Katzav et al. (2008).

DNA isolation was carried out by using GeneJET Genomic DNA Purification Kit (Thermo Scientific, Lithuania). The Supernatant was placed in new tube and stored at $-20^{\circ} \mathrm{C}$.

Campylobacter isolates were identified to the species level by the modification of the method and the primers as described by Wang et al. (2002) and by Katzav et al. (2008). Primers C412F and CampR2 created a 857 bp fragment, which occurred in all Campylobacter spp. A 323-bp amplicon was generated for C. jejuni and a 126-bp amplicon was generated for $C$. coli by using a mixture of primers hybridizing to the C. jejuni (primers CJF and CJR) and the C. coli (primers CCF and CCR). Each PCR mixture contained $2.0 \mu \mathrm{l}$ of $2 \mathrm{mM}$ deoxynucleotide triphosphates (dNTPs) mixture (Thermo Scientific, Lithuania), $2.5 \mu \mathrm{l}$ of 10xPCR buffer, $2.5 \mu \mathrm{l}$ of $25 \mathrm{mM} \mathrm{MgCl}_{2}$, $0.25 \mu$ of Maxima Hot-Start Taq DNA polymerase $5 \mathrm{U} / \mu \mathrm{L}$ (Thermo Scientific, Lithu- 
ania) and $1.0 \mu \mathrm{l}$ of a $100 \mu \mathrm{mol} 1^{-1}$ primer mixture containing C412F, CampR2, C. jejuni and C. coli primers (Thermo Scientific, Lithuania). PCR products were analyzed by gel electrophoresis: $11 \mu \mathrm{l}$ of each PCR product was loaded onto $1.9 \%$ TopVision Agarose gel (Thermo Scientific, Lithuania) containing $6.5 \mu$ l of ethidium bromide solution. The gel was visualized on an UV board. The GeneRuler ${ }^{\mathrm{TM}} 100$ bp DNA Ladder (Thermo Scientific, Lithuania) was used as the molecular size marker.

\section{flaA - RFLP genotyping}

The isolates identified as $C$. jejuni were typed by PCR-RFLP for the flaA gene. A fragment of $1700 \mathrm{bp}$ of the flaA gene was amplified in a PCR reaction by using a pair of specific primers. The PCR was carried out in a $50 \mu$ l (final volume) mixture containing $2.5 \mu \mathrm{l}$ of DNA, $0.5 \mu \mathrm{l}$ of A1 primer and $0.5 \mu \mathrm{l}$ of A2 primer, $5 \mu 1$ of 10xPCR buffer, $5 \mu \mathrm{l}$ of $2 \mathrm{mM}$ dNTP's mix, $3 \mu \mathrm{l}$ of $25 \mathrm{mM} \mathrm{MgCl}_{2}, 0.25 \mu \mathrm{l}$ of Taq DNA polymerase (Thermo Scientific, Lithuania) and $33.25 \mu \mathrm{l}$ of sterilized bidistilled water. The samples were first incubated for $1 \mathrm{~min}$ at $94^{\circ} \mathrm{C}$ and then were cycled 35 times at $94^{\circ} \mathrm{C}$ for $15 \mathrm{~s}$, at $45^{\circ} \mathrm{C}$ for $45 \mathrm{~s}$, and at $72^{\circ} \mathrm{C}$ for 1 min $45 \mathrm{~s}$. Afterwards the samples were incubated at $72^{\circ} \mathrm{C}$ for $5 \mathrm{~min}$ and were maintained at $4^{\circ} \mathrm{C}$ until processed. The PCR product was digested by the incubation at $37^{\circ} \mathrm{C}$ for $3 \mathrm{~h}$ in a reaction mixture containing $21.8 \mu \mathrm{l}$ of sterile bidistilled water, $3 \mu \mathrm{l}$ of buffer, $0.2 \mu \mathrm{l}$ of $\mathrm{DdeI}$ and $5 \mu \mathrm{l}$ PCR product. The digested PCR products were run on $2.5 \%$ agarose at $90 \mathrm{~V}$ for $90 \mathrm{~min}$. The flaA-RFLP gels were visualized on UV board and photographed. The images in TIFF format were performed by using BioNumerics version 7.1 (Applied Maths, Belgium). After the normalization of the pattern, the similarity matrix was calculated by using the Dice similarity coefficient and clustering by the Unweighted Paired Group Method with Arithmetic mean values (UPGMA). The band position tolerance and the optimization coefficient were set to $2.0 \%$. The GeneRuler ${ }^{\mathrm{TM}} 100$ bp plus DNA Ladder (Thermo Scientific, Lithuania) was used as the molecular size marker.

\section{MLST typing}

The target fragments of the housekeeping genes were asp $A$ (aspartase), $g \ln A$ (glutamine synthetase), gltA (citrate synthase), glyA (serine hydroxyl methyl transferase), pgm (phosphor glucomutase), tkt (transketolase), and uncA (ATP synthase alpha subunit). PCR products were amplified with designed primer pairs. The PCR was carried out in a $25 \mu \mathrm{l}$ (final volume) mixture containing $2 \mu \mathrm{l}$ of DNA, $12.5 \mu \mathrm{l}$ DreamTaq Green PCR Master Mix (Thermo Scientific, Lithuania), $2.5 \mu$ l of primer pair mixture and $8 \mu \mathrm{l}$ of sterile bidistilled water. The reaction conditions were: $9 \mathrm{~min}$ at $96^{\circ} \mathrm{C}$ and then were cycled 30 times at $94^{\circ} \mathrm{C}$ for $0.5 \mathrm{~min}$, at $52^{\circ} \mathrm{C}$ for $0.5 \mathrm{~min}$, and at $72^{\circ} \mathrm{C}$ for $1 \mathrm{~min}$. Later the samples were incubated at $72^{\circ} \mathrm{C}$ for $7 \mathrm{~min}$ and were maintained at $4^{\circ} \mathrm{C}$ until processed. The amplicons were examined via gel electrophoresis at $120 \mathrm{~V} 30 \mathrm{~min}$ and PCR product was purified using the GeneJET PCR Purification Kit (Thermo Scientific, Lithuania). The purified PCR products were sent to GATC Biotech AG (Cologne, Germany). Allele numbers were assigned for each housekeeping gene, sequence types (STs) and clonal complexes (CCs) by submitting the DNA sequence to the $C$. jejuni MLST database (http://pubmlst.org/campylobac- 
ter). The obtained sequencing data was analyzed with BioNumerics v 7.1 (Applied Maths, Sint-Martens-Latem, Belgium). For this research was selected the most interesting 60 tested isolates of $C$. jejuni. The number of samples for this research was based on RFLP results. The sixty samples of $C$. jejuni were selected for further analysis by MLST from the most common flaA types.

\section{Results}

\section{Prevalence of Campylobacter in broilers and in environmental sources}

Campylobacter spp. was detected in 12 out of 13 broiler flocks $(92.3 \%)$. In the period of two years we tested 13 broiler flock rotations and collected 1479 samples. In total, 315 (21.3\%) samples were positive for Campylobacter. C. jejuni was identified in $269(85.4 \%)$ samples and $C$. coli in 26 (8.3\%) samples (Table 1). The highest positive samples of Campylobacter were found in broiler cloacae, puddle water and in the litter of additional houses (Table 1). The lowest percentage of Campylobacter was found in the litter of the study house, concentric zones and wild birds (Table 1). However, a few Campylobacter were found in rat fecal samples. We found one positive sample in the anteroom of the study house (Table 1).

Table 1. Prevalence of Campylobacter from broilers and farm environment in Lithuania, 2012-2014

\begin{tabular}{|c|c|c|c|c|}
\hline \multirow[b]{2}{*}{ The type of source } & \multirow{2}{*}{$\begin{array}{l}\text { No. of tested } \\
\text { samples }\end{array}$} & \multicolumn{3}{|c|}{ No. (\%) of positive samples } \\
\hline & & $\begin{array}{c}\text { other } \\
\text { Campylobacter spp. }\end{array}$ & C. jejuni & C. coli \\
\hline Litter of the study broiler house & 81 & $2(11.8)$ & $12(70.6)$ & $3(17.6)$ \\
\hline Litter of additional broiler houses (3) & 229 & $1(1.8)$ & $42(77.8)$ & $11(20.4)$ \\
\hline Anteroom of study house & 67 & 0 & $1(100.0)$ & 0 \\
\hline Zones & 305 & 0 & $30(93.8)$ & $2(6.2)$ \\
\hline Puddles & 166 & $12(25.5)$ & $35(74.5)$ & 0 \\
\hline Wild birds & 56 & 0 & $6(100.0)$ & 0 \\
\hline Rats & 40 & $2(100.0)$ & 0 & 0 \\
\hline Wild animals faeces & 12 & 0 & $1(100.0)$ & 0 \\
\hline Flies & 138 & 0 & 0 & 0 \\
\hline Broiler cloacae & 402 & $3(1.9)$ & $142(91.6)$ & $10(6.5)$ \\
\hline Tap water & 50 & 0 & 0 & 0 \\
\hline
\end{tabular}

Campylobacter counts (cfu/g) were calculated before every slaughter of the flock. The data shows that a higher Campylobacter spp. number was established in crop 4 during the spring period of March-April $\left(6.86 \log _{10} \mathrm{cfu} / \mathrm{g}\right)$ and in crop 9 during the winter time in December-January $\left(6.78 \log _{10} \mathrm{cfu} / \mathrm{g}\right)$ (Figure 1). The lowest number of Campylobacter was in crop 2 (November-December) and crop 7 (September-October). 


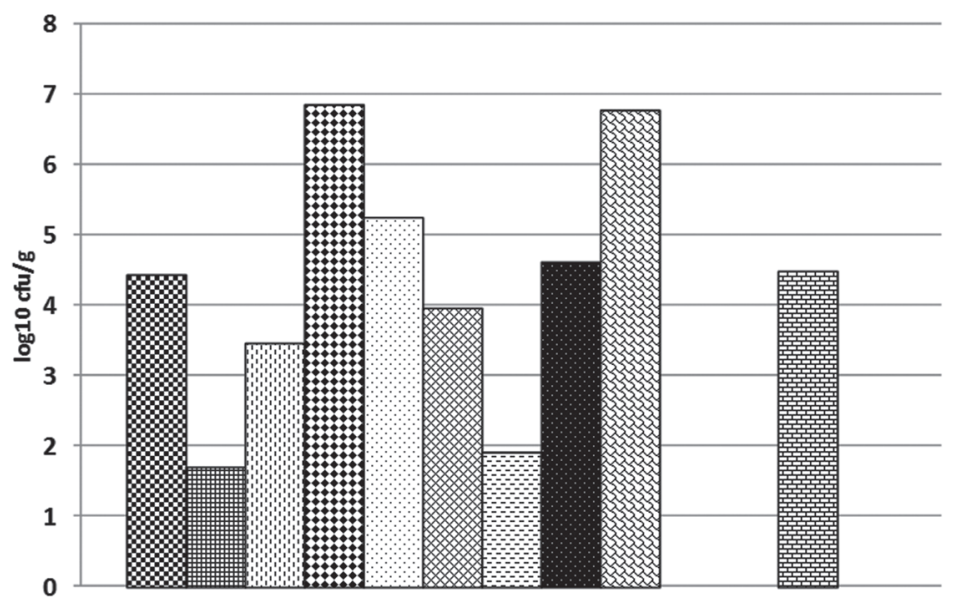

界rop 1 (9-10)

田 Crop 2 (11-12)

머 Crop 3 (1-2)

to Crop 4 (3-4)

๑Crop 5 (5-6)

因 Crop 6 (7-8)

중 Crop 7 (9-10)

Crop $8(10-11)$

$\checkmark$ Crop 9 (12-1)

- Crop 10 (2-3)

$\square$ Crop 11 (4-5)

国 Crop 12 (6-7)

Figure 1. The numbers of Campylobacter spp. before the slaughtering at farm

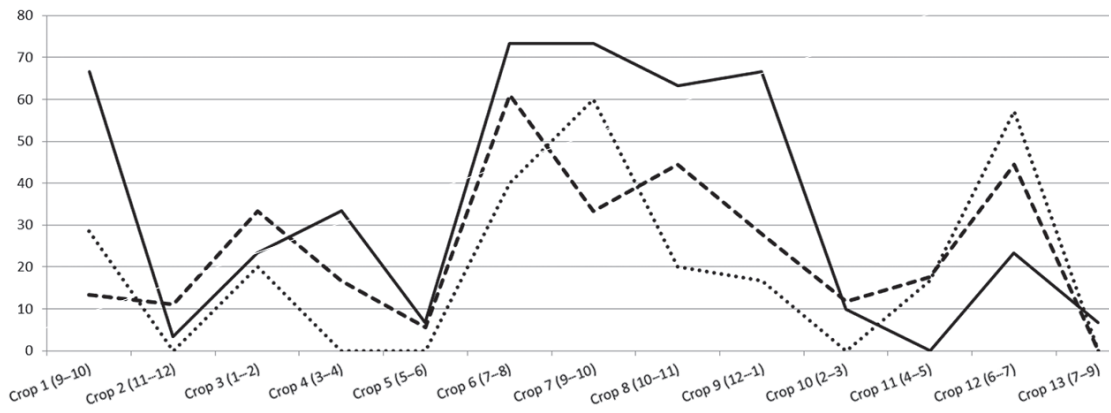

..... Litter in study house $\quad$ - - Litter in additional houses $\quad$ Broiler cloaca

Figure 2. Campylobacter spp. prevalence in litter and broiler cloaca

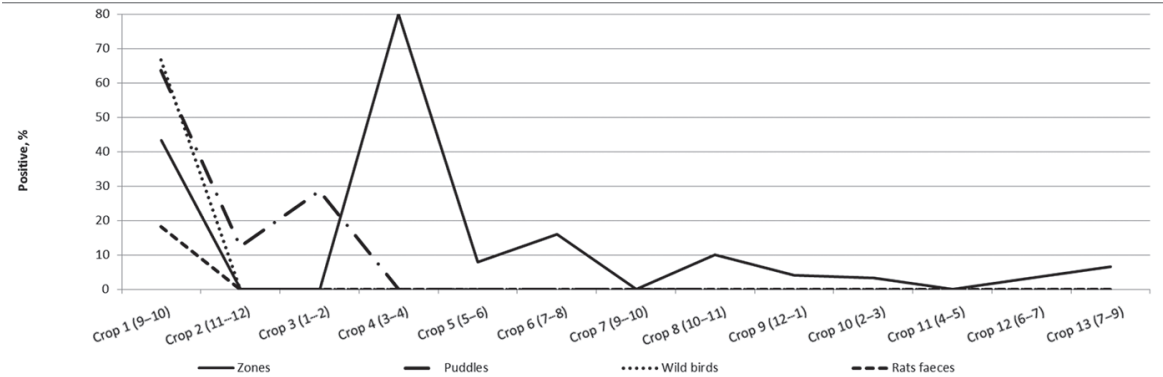

Figure 3. Campylobacter spp. prevalence in broiler environment 


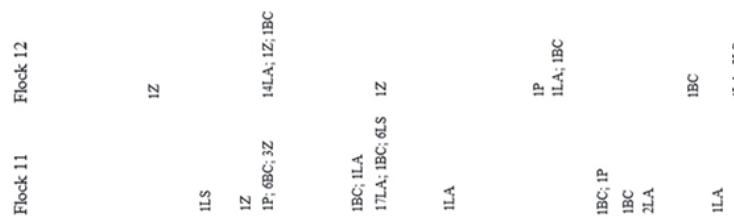

$\stackrel{\frac{7}{8}}{\frac{8}{4}}$

$\frac{9}{8}$

尊的

魚N

正

范首

芯总

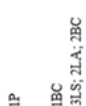

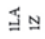

$\leqq$

a
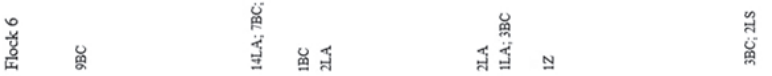

尊

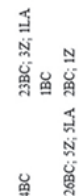

竞

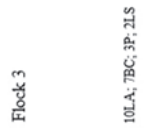

点

窇需曾

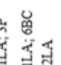

运运

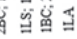

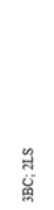

Nㅜㅇ

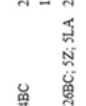

$\overrightarrow{\frac{.}{8}} \dot{a}$

a
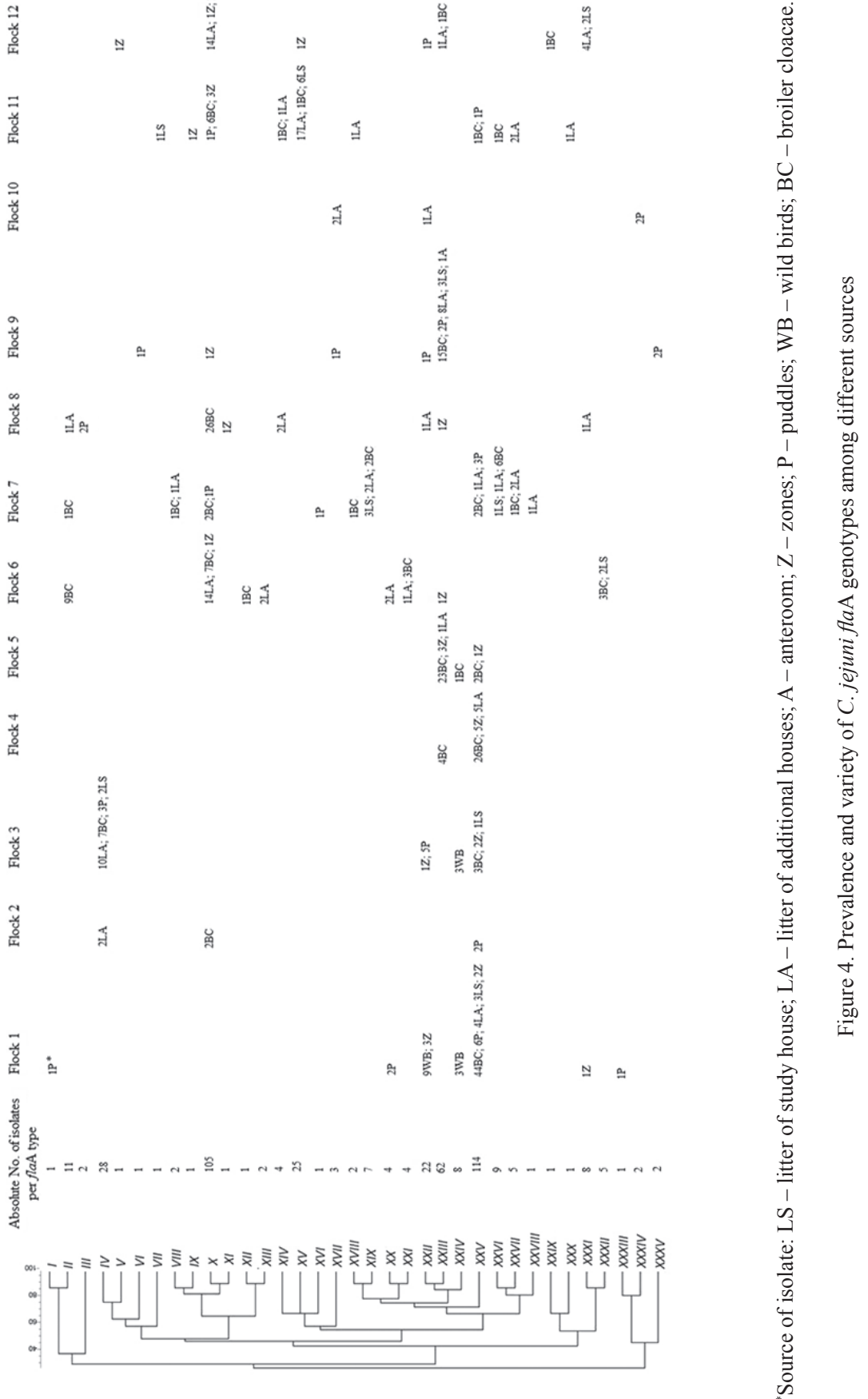
The prevalence of Campylobacter in broiler cloaca samples increased in September-October (crop 1) and also from July to August (crop 6 and crop 10) (Figure 2). The results showed the peaking in July-August (crop 6) and in September-October (crop 7), but in April-May (crop 11) no positive samples were found. A higher increase was detected in the litter of study house samples in September-October (crop 7) and June-July (crop 12). In crops 2, 4, 5, 10 and 13 we have not found positive samples. The positivity of the samples from litter of the additional houses showed the increase in July-August (crop 6).

Figure 3 shows positive samples from the environment sources. The highest Campylobacter contamination as seen in the samples was collected from the zones, in the puddle and in wild birds. We have to assume that the peak in the zones was reached in March-April (crop 4). The highest prevalence in puddles was in September-October when crop 1 was raised. From March no positive samples from puddles were found. The similar results could be seen in other sources (wild birds and rats feces).

\section{Genotype diversity of $\boldsymbol{C}$. jejuni isolates}

The flaA-RFLP typing of $448 C$. jejuni isolates resulted in 35 different $f l a A$ genotypes (Figure 4). The most frequent flaA genotypes were $\mathrm{X}$ and $\mathrm{XXV}$, these genotypes were found in the water of puddles, the litter of study and additional houses, broiler cloaca and the zones. Both genotypes were detected in 7 flocks. We distinguished 3 groups of the genotypes. To the first group belong $C$. jejuni genotypes XII and XXVIII and they were dominant just in broiler cloacal samples in flocks 6 and 12. The second group comprises genotypes I, III, V-VII, IX, XI, XIII, XVI, XVII, XIX, XXII, XXIX-XXXI, XXXIII-XXXV which were found just in the farm environment and in the litter of additional houses. And the third group genotypes II, IV, VIII, X, XIV, XV, XVIII, XX, XXI, XXIII-XXVII and XXXII were dominant in all sources.

\section{Diversity and distribution of STs and CCs among broilers and in environ- mental sources}

Among 60 C. jejuni isolates which were included in MLST analysis, 20 distinct STs were identified. Among all the STs 9 isolates (15\%) were assigned to 2 different STs (ST-7413 and ST-4800), which could not be assigned to any of the known CCs registered at PubMLST database (http://pubmlst.org/campylobacter) (Table 2). The most common CCs were ST-21 CC (20\%) and ST-179 CC (16.6\%). The six most predominant STs (ST-21, ST-4447, ST-257, ST-5, ST-51 and ST-4800) represented approximately half of the isolates (33 out of 60 isolates, or 55\%). The ST-21 complex was the most widely distributed clonal complex and was present in 5 of 7 different isolation sources (Table 3). The ST-52 and ST-464 complexes were the least widely distributed. ST-179 complex was predominant in puddles as compared with other CCs. The wild birds had one clonal complex ST-179, which was predominant as compared with other sources. Conversely, unassigned complexes were predominant in broiler cloaca. Two clonal complexes (ST-257 and ST-353) were found in the same sources (the broiler cloaca, the zones and the litter of the study house and ad- 
ditional houses). In this study ST-21, ST-257 and ST-353 clonal complexes were common in broiler cloaca, the zones and the litter of all houses. Two sources (the zones and the litter of additional houses) had 8 out of 11 different clonal complexes and six clonal complexes were common to both isolation sources. The population structure of the isolates was also evaluated with a minimum-spanning tree (Figure 5) using the allelic date with the program Bionumerics V 7.0. The phylogenetic analysis showed that the most STs are common in various sources. Only few STs are specific and found in these sources, such as ST-464 established from anteroom; ST-5013 and ST-2066 established from the litter of additional houses and ST-7413 and ST-5777 established from the broiler cloaca.

Table 2. Distribution of 60 C. jejuni isolates among CCs and STs

\begin{tabular}{|c|c|c|}
\hline $\mathrm{CC}$ & ST & No. of $C$. jejuni isolates \\
\hline \multirow[t]{4}{*}{21} & 19 & 1 \\
\hline & 21 & 6 \\
\hline & 376 & 3 \\
\hline & 2857 & 2 \\
\hline 48 & 429 & 3 \\
\hline 52 & 2066 & 1 \\
\hline \multirow[t]{3}{*}{179} & 220 & 2 \\
\hline & 2209 & 2 \\
\hline & 4447 & 6 \\
\hline \multirow[t]{2}{*}{257} & 257 & 5 \\
\hline & 2254 & 2 \\
\hline \multirow[t]{2}{*}{353} & 5 & 5 \\
\hline & 5011 & 1 \\
\hline 443 & 51 & 6 \\
\hline 464 & 464 & 1 \\
\hline 607 & 607 & 2 \\
\hline \multirow[t]{2}{*}{828} & 829 & 2 \\
\hline & 5777 & 1 \\
\hline \multirow[t]{2}{*}{ Unassigned } & 7413 & 4 \\
\hline & 4800 & 5 \\
\hline
\end{tabular}

Table 3. Frequency and distribution of clonal complexes among broilers and their environmental sources of C. jejuni

\begin{tabular}{l|c|c|c|c|c|c|c|c|c|c|c}
\hline \multirow{2}{*}{\multicolumn{1}{c}{ Source (n) }} & \multicolumn{10}{c}{ Clonal complex } \\
\cline { 2 - 11 } & 21 & 48 & 52 & 179 & 257 & 353 & 443 & 464 & 607 & 828 & Unassigned \\
\hline Broiler cloacae (17) & 4 & - & - & - & 2 & 1 & 1 & - & - & 2 & 7 \\
Puddles (7) & 2 & 1 & - & 3 & - & - & 1 & - & - & - & - \\
Wild birds feces (5) & - & - & - & 5 & - & - & - & - & - & - & - \\
Zones (11) & 1 & - & - & 2 & 2 & 1 & 2 & - & 1 & 1 & 1 \\
Litter of the study house (6) & 3 & - & - & - & 1 & 1 & - & - & - & - & 1 \\
Litter of additional houses (13) & 2 & 2 & 1 & - & 2 & 2 & 2 & - & 1 & 1 & - \\
Anteroom of study house (1) & - & - & - & - & - & - & - & 1 & - & - & - \\
\hline
\end{tabular}




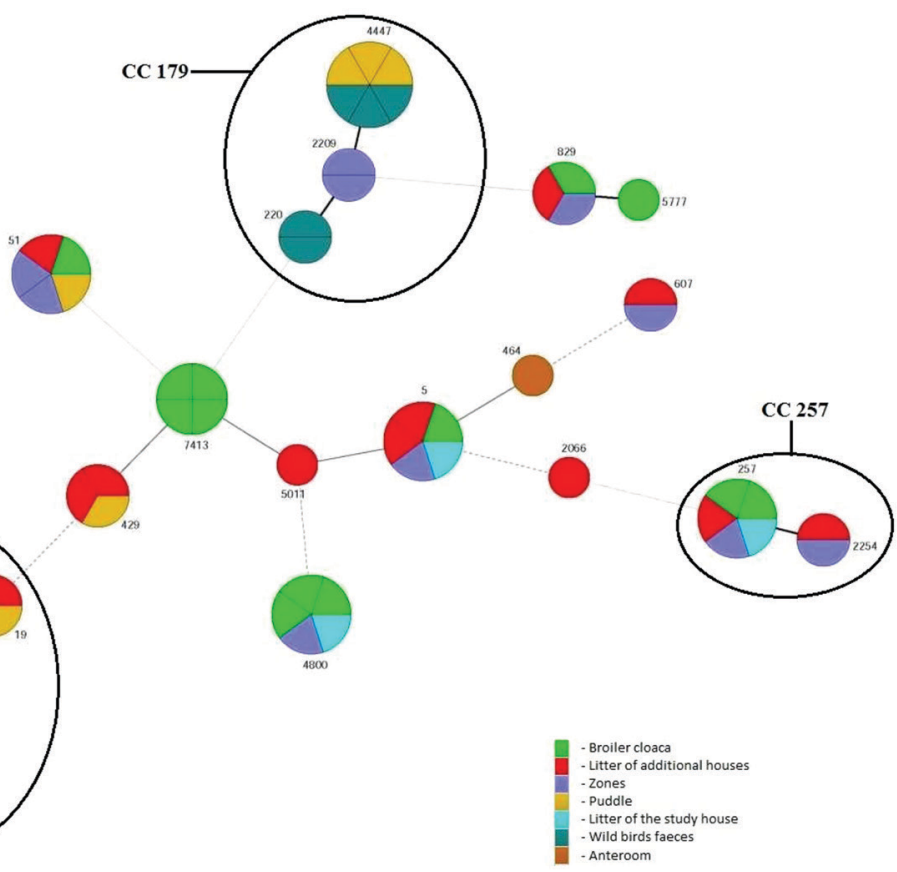

Figure 5. Phylogenetic analysis of C. jejuni isolates from broilers and their environment by MLST. Each circle and number by the circle represents one ST: circles of increased diameter represent higher strain numbers within one ST, different colours indicate strain source. Numbers in bold by the circle represent three of the most prevalent CCs revealed in this study

\section{Discussion}

The horizontal transmission from the environment is considered to be the most frequent source of Campylobacter spp. to broilers. It is widely accepted that the horizontal transmission within a flock occurs rapidly in case individual birds are recolonized by Campylobacter (Carrillo et al., 2004; Horrocks et al., 2009). Once established it is very difficult to eliminate. High flock size, environmental water supplies, litter, insects, wild birds, rodents, fecal contact, personnel and other animals, may increase the risk of colonization and dissemination (Adkin et al., 2006; Horrocks et al., 2009).

This study shows the higher prevalence (92.31\%) of Campylobacter spp. in broiler flocks compared to the previous study of Kudirkienè et al. (2010). In neighboring Poland also most of the flocks were positive (96.5\%) (Wieczorek and Osek, 2015). Furthermore, the investigation as reported by Hungary and the United Kingdom showed very high proportions of positive samples (from $74.2 \%$ to $80 \%$ ) (EFSA, 2015) as well. Our study has revealed that the environmental sources carrying Campylobacter in the poultry farm were associated with positive broiler flocks. Fi- 
gure 2 shows that Campylobacter prevalence in broilers and in the litter are related. If the litter is positive to Campylobacter then the flock is positive too. The higher prevalence of Campylobacter in litter and in broilers was found from July to January. Dry and aerobic conditions of clean fresh litter are considered harmful to C. jejuni as reported by Newell and Fearnley (2003) and Hutchinson et al. (2005). On the other hand, litter can be contaminated by broiler fecal droppings, which favors pathogen transmission through the flock. Dirty contaminated litter can spread in the poultry farm and scatter the microorganism in the environment.

This study has showed that in the period of September-October (crop 1) high Campylobacter spp. prevalence was found in wild birds (66.6\%), however, from December we did not find any positive samples. In contrast, other studies showed the high Campylobacter spp. frequency in wild birds (Brown et al., 2004; Hughes et al., 2009). In general, Campylobacter can survive in water, for this reason puddles were found to be positive, but just from September to April. Later we did not detect positive samples. Maybe the positivity of puddles may reflect the general level of cleanliness and contamination of other environmental sources in the farm.

The typing of Campylobacter isolates from broilers and their environment provides epidemiological information which is needed for the infection control and the transmission. flaA-RFLP typing and MLST were used to explain genetic diversity among isolates.

Results from $f l a A-R F L P$ typing showed that most of the genotypes were the same in broilers and in their environment. These genotypes were found first in the environment, later in the litter and next in the broilers. Such phenomenon indicates that the bacteria move from the environment to the flock. There are several potential sources of Campylobacter that show a temporal correlation with the flock becoming Campylobacter positive, such as rodents (Umali et al., 2012; Nkogwe et al., 2011), humans (Messens et al., 2009; Ridley et al., 2011) and water (Patriarchi et al., 2011), which may indicate that these potential sources are actually becoming contaminated by the broiler flock as it is colonized. Catching crews and independent operators (Young et al., 2010) were identified as a potential source of new genotypes in the flock during late grow-out when Campylobacter is shedding in the contaminated flock peaks in this way increasing the chances of within-farm and industry-wide Campylobacter dissemination. During the analysis we found only one positive sample from the anteroom in flock 9 and it belongs to XXIII flaA type. The contamination of the anteroom floor may actually suggest the possible infection of the flock by the farmer, visitors and equipment. Unsuitable hygiene practices at the farm level, especially poor cleaning and disinfection of the house and not dedicated protective clothing, could be a major reason of Campylobacter contamination persistence in poultry flocks as was stated by Allen and Newell (2005). Two flocks (7 and 11) had 10 different genotypes, which were found in broilers and in their environment. These flocks were raised in different seasons, flock 7 was raised in April-May and flock 11 was raised in the period of August-September. However, both seasons had the same temperature $\left(7-16^{\circ} \mathrm{C}\right)$. In flocks $2,3,4,9,10$ we found less different genotypes. All these flocks were raised in winter and in spring and the temperature was below $0^{\circ} \mathrm{C}$. The conclusion can be made that the temperature has the effect on Campylobacter 
survival mechanism. Many studies showed that Campylobacter is sensitive to low temperatures (Silva et al., 2011; Vashin and Stoyanchev, 2011) and, therefore, has less chances to survive in the environment and spread to the flocks. A similar pattern with high diversity of types in warm season and lower diversity of types in cold season has been reported in other studies as well (Hiett et al., 2002; Kudirkienè et al., 2010). The most dominant is flaA type XXV, which was found in 7 flocks out of 12. These flocks were raised in different seasons (spring, autumn and winter). We could assume that this flaA type is more tolerant to the environmental stresses. Duffy et al. (2015) and Allen et al. (2007) also suggested that some genotypes could be more tolerant to the environmental stresses in poultry processing.

MLST is an important technique which was invented to study population of Campylobacter spp. (Dingle et al., 2001; Levesque et al., 2008). Molecular typing has previously been used to infer the source of campylobacteriosis and the overlap in the genotypes of Campylobacter species present in humans, broilers and other sources (Revez et al., 2014; Ramonaite et al., 2014; Llarena et al., 2014; Zhang et al., 2015). In the study for MLST analyses the isolates have been selected randomly. The isolates were selected from flaA types, which were isolated from various sources. 8 isolates were selected from IV flaA type, 1 - from V flaA type, 6 - from VII flaA type, 7 - from VIII flaA type, 1 - from IX flaA type, 2 - from X flaA type, 3 - from XI flaA type, 2 - from XII flaA type, 5 - from XVI flaA type, 3 - from XVIII flaA type, 5 - from XX flaA type, 3 - from XXI flaA type, 2 - from XXIV flaA type, 2 - from XXV flaA type, 8 - from XXIX flaA type, 1 - from XXXII flaA type and 1 - from XXXIII flaA type. We analyzed the ST diversity and the population structure of 60 C. jejuni isolates from different sources. Twenty STs were defined from 60 isolates, which were classified into 10 clonal complexes. Many previous studies have shown that the main clonal complex varies in different regions and countries but CCs, such as ST-45, ST-21, ST-48 and ST-353 are dominant STs that contain the largest number of isolates (Petkovic et al., 2009; Ramonaite et al., 2014; Zhang et al., 2015). In the study out of 10 identified CCs, five (ST-21 CC, ST-179 CC, ST$443 \mathrm{CC}$, ST-353 CC and ST-257 CC) were the most prevalent and $41(68.3 \%)$ of our examined isolates were labelled to these clonal complexes. At least three individual isolates were assigned to one of these five clonal complexes. One of these clonal complexes (ST-179 CC) was specific to wild birds and 5 C. jejuni isolates $(8.3 \%)$ were labelled to this clonal complex. ST-21 CC was dominant in broilers $(6.7 \%)$ and in the litter of the study house (5\%). All these clonal complexes were identified from different sources: $12(20 \%)$ of the tested isolates were assigned to ST-21 CC, $10(16.7 \%)$ to ST-179 CC, $7(11.7 \%)$ to ST-257 CC, $6(10 \%)$ to ST-353 CC and $6(10 \%)$ to ST-443 CC. The remnant five clonal complexes were identified sporadically among tested $C$. jejuni isolates.

Some studies have reported that $C$. jejuni strains which belong to the ST-21 complex can colonize a multiplicity of hosts and they are one of the most common CCs that infect humans (Cody et al., 2013; El-Adawy et al., 2013; Ramonaite et al., 2014; Zhang et al., 2015). Thus, the study showed that ST-21 CC contains four strains from broilers, three strains from the litter of the study house, two strains from the puddle and the litter of additional houses and 1 strain from the zones. This clonal complex 
is associated with poultry and environment. A similar pattern has been reported in other studies (de Haan et al., 2010; Magnusson et al., 2011; Zhang et al., 2015) but in Ramonaite et al. (2014) study they have not identified ST-21 CC among C. jejuni isolates from environmental sources.

ST-179 CC was the most common complex in the feces of wild birds, also this complex was found in the puddle and in the zones (environment). The prevalence of this clonal complex in wild birds and the puddle was previously registered by Ramonaite et al. (2014) and Meinersmann et al. (2013). In our study three STs (220, $2209,4447)$ assigned to this complex were identified. According to the PubMLST these STs are found in human stool, sand, environmental water, wild birds and in chickens.

ST-257 and ST-353 clonal complexes are also described in other studies (Colles et al., 2003; Zhang et al., 2015; El-Adawy et al., 2013). We assigned two STs (257, 2254 ) to ST-257 complex and one ST (5011) to ST-353 complex, all strains were isolated from broilers, litter and zones. Interestingly, these STs according to PubMLST are found in human stool, turkey, chicken meat, but not in environmental sources.

According to PubMLST ST-51 of ST-443 complex is found in various sources, such as dog, duck, cattle, human stool, chicken meat and turkey. In our study, we have found ST-51 in the broiler, the puddle, the zones and in the litter of additional houses. However, in other studies this clonal complex was found sporadically (Zhang et al., 2015; Ramonaitè et al., 2014).

The results of our study indicate that Campylobacter spp. is widespread in broilers and in environmental sources, with the variation of genotypes. In conclusion, the most important thing is to prevent Campylobacter spread in the environment to enter the broiler house. We found STs among C. jejuni isolates from the litter, wild birds, the zones and the puddles, thus, the fact reveals that these Campylobacter sources are not enough investigated. Further studies are needed to analyze all possible sources which induce the spread of Campylobacter. Furthermore, isolates have to be characterized by genome sequencing for a better understanding of campylobacteriosis epidemiology.

\section{Acknowledgements}

The work was financially supported by the 7BP ERA-NET project EMIDA of the Ministry of Agriculture of the Republic of Lithuania (project CAMCHAIN TM-12/1).

\section{References}

A d kin A., Hartnet t E., J ord a n L., N e w e 11 D., D a vi d s o n H. (2006). Use of systematic review to assist the development of Campylobacter control strategies in broilers. J. Appl. Microbiol., 100: 306-315.

A 11 e n V.M., N e w e 11 D.G. (2005). Evidence for the effectiveness of biosecurity to exclude Campylobacter from poultry flocks. Food Standard Agency Report, Commissioned project MS0004, http:// www.food.gov.uk/multimedia/pdfs/biocampy.pdf

A 11 e n V.M., B u 11 S.A., C orry J.E., D o m ingue G., Jorgen s e n F., Fros t J.A., Wh y te R., 
Gonzalez A., Elvis s N., Humphrey T.J. (2007). Campylobacter spp. contamination of chicken carcasses during processing in relation to flock colonisation. Int. J. Food Microbiol., 113: 54-61.

B rown P.E., Chri s te n s e n O.F., C 1 o u g h H.E., D ig g l e P.J.,H a rt C.A., Ha z e 1 S., K e mp R., L e a therbarrow A.J.H., Moore A., Sutherst J., Turner J., Williams N.J., Wright E.J., French N.P. (2004). Frequency and spatial distribution of environmental Campylobacter spp. Appl. Environ. Microbiol., 70: 6501-6511.

Bunevičienè J., Kudirkiene E., Ramonaitė S., Malakauskas M. (2010). Occurrence and numbers of Campylobacter spp. on wings and drumsticks of broiler chickens at the retail level in Lithuania. Vet. Med. Zoot., 50: 9-14.

Carrillo C.D., Tabo ad a E., N a sh J.H.E., Lanthier P., Kelly J., La u P.C., Verhulp R., Mykytczuk O., Sy J., Findlay W.A., Amoako K., Gomis S., Wills on P., Aus t in J.W., P otter A., B a bi u k L., A 11 a n B., S z y man s k i C.M. (2004). Genome-wide expression analyses of Campylobacter jejuni NCTC11168 reveals coordinate regulation of motility and virulence by flhA. J. Biol. Chem., 279: 20327-20338.

Cody A.J., McCarthy N.D., Jansen van Rensburg M., Is inkaye T., B entley S.D., P arkhill J., Dingle K.E., B ow ler I.C., Jolley K.A., Ma id en M.C. (2013). Real-time genomic epidemiological evaluation of human Campylobacter isolates by use of whole-genome multilocus sequence typing. J. Clin. Microbiol., 51: 2526-2534.

Colle s F.M., J o nes K., H a r d ing R.M., Ma i d e n M.C.J. (2003). Genetic diversity of Campylobacter jejuni isolates from farm animals and the farm environment. Appl. Environ. Microbiol., 69: $7409-7413$.

D e Haan C.P., Kivistö R., Hakkinen M., Rautelin H., Hänninen M.L. (2010). Decreasing trend of overlapping multilocus sequence types between human and chicken Campylobacter jejuni isolates over a decade in Finland. Appl. Environ. Microbiol., 76: 5228-5236.

D ingle K.E., Colle s F.M., Ware ing D.R., U re R., F ox A.J., B olt o n F.E., B o o t s m a H.J., W i 11 e m s R.J., U rw in R., M a i d e n M.C. (2001). Multilocus sequence typing system for Campylobacter jejuni. J. Clin. Microbiol., 39: 14-23.

D uffy L.L., B l a ckall P.J., C obbold R.N., F e g a n N. (2015). Mapping the carriage of flaArestriction fragment length polymorphism Campylobacter genotypes on poultry carcasses through the processing chain and comparison to clinical isolates. Food Microbiol., 48: 116-122.

El-Adawy H., Hotzel H., Tomaso H., Neubauer H., Taboada E.N., Ehricht R., H a fe z H.M. (2013). Detection of genetic diversity in Campylobacter jejuni isolated from a commercial turkey flock using flaA Typing, MLST analysis and microarray assay. PLOS One., 8(2): $1-11$.

E 11 is - I vers en J., R i d d l e y A., M o r r is V., S o w a A., H a r r is J.,A t t e r bury R., S p a r k s N., A 11 e n V. (2012). Persistent environmental reservoirs on farms as risk factors for Campylobacer in commercial poultry. Epidemiol Infect., 140: 916-924.

European Food Safety Authority (EFSA) (2015). The European Union summary report on trends and sources of zoonoses, zoonotic agents and food-borne outbreaks in 2013. EFSA J., 13: 1-162.

H i e t t K.L., S t e r n N.J., F e d ork a - C r a y P., C ox N.A., M u s g rove M.T., L a d e l y S. (2002). Molecular subtype analyses of Campylobacter spp. from Arkansas and California poultry operations. Appl. Environ. Microbiol., 68: 6220-6236.

Horrocks S.M., A n d e r s on R.C., N i el s be t D.J., R i c k e S.C. (2009). Incidence and ecology of Campylobacter jejuni and coli in animals. Anaerobe, 15: 18-25.

Hughes L.A., B ennett M., Coffey P., E 11 i ott J., Jones T.R., Jones R.C., Lahuerta - Marin A., Leatherbarrow A.H., McNiffe K., Norman D., Williams N.J., Chantrey J. (2009). Molecular epidemiology and characterization of Campylobacter spp. isolated from wild bird populations in northern England. Appl. Environ. Microbiol., 75: 3007-3015.

Hutchins on M.L., Walters L.D., Avery S.M., Munro F., Moore A. (2005). Analyses of livestock production, waste storage and pathogen levels and prevalences in farm manures. Appl. Environ. Microbiol., 71: 1231-1236.

ISO/TS 10272-2:2006. Microbiology of food and animal feeding stuffs. Horizontal method for detection and enumeration of Campylobacter spp. Part 2: Colony-count technique. 
Jak opanec I., B orgen K., Vold L., Lund H., Forseth T., Hannula R., Nygard K. (2008). A large waterborne outbreak of campylobacteriosis in Norway: the need to focus on distribution system safety. BMC Infect Dis., 8: 1-11.

Katzav M., I s ohanni P., Lund M., Hakkinen M., Lyhs U. (2008). PCR assay for the detection of Campylobacter in marinated and non-marinated poultry products. Food Microbiol., 25: 908-914.

Korczak B.M., Zurfluh M., Emler S., Kuhn-Oertli J., Kuhnert P. (2009). Multiplex strategy for multilocus sequence typing, fla typing, and genetic determination of antimicrobial resistance of Campylobacter jejuni and Campylobacter coli isolates collected in Switzerland. J. Clin. Microbiol., 47: 1996-2007.

Kudirkienė E., Malakauskas M., Malakauskas A., Bojesen A.M., Olsen J.E. (2010). Demonstration of persistent strains of Campylobacter jejuni within broiler farms over a 1-year period in Lithuania. J. Appl. Microbiol., 108: 868-877.

Lar sen M.V., C o s entino S., R a s mus sen S., Fri is C., H a s man H., Marvig R.L., Je 1 s bak L., Sicheritz-Ponte'n T., Ussery D.W., A a restrup F.M., Lund O. (2012). Multilocus sequence typing of total-genome sequenced bacteria. J. Clin. Microbiol., 50: 1355-1361.

L e ve s que S., Fros t E., A r b e it R.D., M i c ha u d S. (2008). Multilocus sequence typing of Campylobacter jejuni isolates from humans, chickens, raw milk, and environmental water in Quebec, Canada. J. Clin. Microbiol., 46: 3404-3411.

L l a ren a A.K., Hun ea u A., Hakk in en M., Hänn inen M.L. (2014). Predominant Campylobacter jejuni sequence types persist in Finnish chicken production. PLOS One., 10(2): 1-18.

Magnus son S.H., Guðmundsdottir S., Reynisson E., Runars son A.R., Harðar dottir H., Gunnarson E., Georgs son F., Reiersen J., Marteinsson V.T. (2011). Comparison of Campylobacter jejuni isolates from human, food, veterinary and environmental sources in Iceland using PFGE, MLST and fla-SVR sequencing. J. Appl. Microbiol., 111: 971-981.

Me in ersmann R.J., B errang M.E., Litt le E. (2013). Campylobacter spp. recovered from the upper Oconee river watershed, Georgia in a 4-year study. Microb. Ecol., 65: 22-27.

Messens W., Herman L., De Zutter L., Heyndrickx M. (2009). Multiple typing for the epidemiological study of contamination of broilers with thermotolerant Campylobacter. Vet. Microbiol., 138: 120-131.

M o ffat t C.R., C a m e r o n S., M i c k a n L., G i vn e y R.C. (2010) Campylobacter jejuni gastroenteritis at an Australian boarding school: consistency between epidemiology, flaA typing, and multilocus sequence typing. Foodborne Pathog. Dis., 7: 1285-1290.

Newe 11 D.G., Fe arnley C. (2003). Sources of Campylobacter colonization in broiler chickens. Appl. Environ. Microbiol., 69: 4343-4351.

Nkogwe C., Ralet tobana J., Stewart-Johnson A., Suepaul S., Adesiyun A. (2011). Frequency of detection of Escherichia coli, Salmonella spp., and Campylobacter spp. in the faeces of wild rats (Rattus spp.) in Trinidad and Tobago. Vet. Med. Int., 686923: 1-7.

Patriarchi A., Fox A., Maunsell B., F anning S., B olt on D. (2011). Molecular characterization and environmental mapping of Campylobacter isolates in a subset of intensive poultry flocks in Ireland. Foodborne Pathog. Dis., 8: 99-108.

Petkovic J., Rudi K., Buncic O., Kat i c V., Lepsanovic Z. (2009). Multilocus sequence typing for investigation of diversity of Campylobacter jejuni strains from humans and environment in Norway. Acta Vet. Hung., 57: 1-11.

Ramonaitè S., Rokaitytė A., Tamulevičienè E., Malakauskas A., Malakaus k a s M. (2013). Prevalence, quantitative load and genetic diversity of Campylobacter spp. in dairy cattle herds in Lithuania. Acta Vet. Scand., 55: 1-6.

Ramonaitė S., Kudirkienè E., Tamulevičienè E., Levinienè G., Malakausk a s A., Gölz G., Alter T., Malakauskas M. (2014). Prevalence and genotypes of Campylobacter jejuni from urban environmental sources in comparison with clinical isolates from children. J. Med. Microbiol., 63: 1205-1213.

Revez J., Llarena A.K., Schott T., Kuusi M., Hakkinen M., Kivisto R., Hanninen M.L., Ross i M. (2014). Genome analysis of Campylobacter jejuni strains isolated from a waterborne outbreak. BMC Genomics, 15: 1-8. 
Ri chard son G., Thom a s D.R., S m i th R.M.M., N e ha u 1 L., R i b e ir o C.D., B rown A.G., S a 1 m o n R.L. (2007). A community outbreak of Campylobacter jejuni infection from a chlorinated public water supply. Epidemiol. Infect., 135: 1151-1158.

Ridley A.M., Morris V.K., Cawthraw S.A., E 11 is - Iversen J., Harris J.A., Kenned y E.M., N e w e 11 D.G., A 11 e n V.M. (2011). Longitudinal molecular epidemiological study of thermophilic Campylobacters on one conventional broiler chicken farm. Appl. Environ. Microbiol., 77: 98-107.

Silva J., Leite D., Fernandes M., Mena C., Gibbs P.A., Teix e ir a P. (2011). Campylobacter spp. as a food borne pathogen: a review. Front Microbiol., 2: 1-12.

Umali D.V., Lapuz R.R.S.P., Suzuki T., Shirota K., K a toh H. (2012). Transmission and shedding patterns of Salmonella in naturally infected captive wild roof rats (Rattus rattus) from a Salmonella-contaminated layer farm. Avian Dis., 56: 288-294.

Va s h in I.T., S t o y a $\mathrm{n} \mathrm{h} \mathrm{e} \mathrm{v} \mathrm{T.T.} \mathrm{(2011).} \mathrm{Influence} \mathrm{of} \mathrm{temperature} \mathrm{on} \mathrm{Campylobacter} \mathrm{jejuni} \mathrm{survival}$ rates in pork meat. J. Vet. Med., 14: 25-30.

W a ng R.F., S l a v i c M.F., C a o W.W. (2002). A rapid PCR method for direct detection of low numbers of Campylobacter jejuni. J. Rapid Meth. Aut. Mic., 1: 101-108.

W i e c z o rek K., O s e k J. (2015). Poultry flocks as a source of Campylobacter contamination of broiler carcasses. Pol. J. Vet. Sci., 18: 101-106.

Will i a m s J.K., S a it L.C., Cogan T.A., Jorgensen F., Grogono-Thomas R., Humphrey T.J. (2012). Enrichment culture can bias the isolation of Campylobacter subtypes. Epidemiol. Infect., 140: 1227-1235.

Young I., Rajic A., Letellier A., Cox B., Les lie M., S anei B., Mc Ewen S.A. (2010). Knowledge and attitudes toward food safety and use of good production practices among Canadian broiler chicken producers. J. Food Prot., 73: 1278-1287.

Zhang G., Zhang X., Hu Y., Ji a o X., Huang J. (2015). Multilocus sequence types of Campylobacter jejuni isolates from different sources in Eastern China. Curr. Microbiol., 71: 341-346.

Received: 6 XI 2018

Accepted: 3 I 2020 